\title{
A physiological model for the control of erythromycin production in batch and cyclic fed batch culture
}

\author{
M. E. Bushell, J. Smith† and H. C. Lynch‡ \\ Author for correspondence: M. E. Bushell. Tel: +44 1483 259277. Fax: +44 1483300374. \\ e-mail: m.bushell@surrey.ac.uk
}

Microbial Products Laboratory, Biological Sciences, University of Surrey, Guildford, Surrey, GU2 5XH, UK

\begin{abstract}
Reported differences in antibiotic production dynamics resulting from altering the growth-limiting nutrient (growth-dissociated production in carbon-limited culture and apparent growth-associated production in nitrogen-limited culture) are due to the different effects on growth kinetics. The substrate affinity for nitrate is significantly lower than that for glucose, resulting in nitrogen limitation effectively occurring throughout the culture. Glucose limitation occurs later in the culture, coinciding with the induction of antibiotic production. Induction occurs at the start of nitrogen-limited culture so that production appears to be growth-associated. Evidence that this hypothesis is consistent with production kinetics in cyclic fed batch culture was also obtained.
\end{abstract}

Keywords: erythromycin, physiological control model, cyclic fed batch culture

\section{INTRODUCTION}

Despite the established societal and economic significance of antibiotics, generally accepted physiological control concepts have only emerged comparatively recently (reviewed by Bibb, 1996). Antibiotics, in common with many other bioactive microbial metabolites, are classified as secondary metabolites. Such compounds have been described as being produced after the culture growth rate has decayed during batch culture (e.g. Demain et al., 1983). This coincides with the exhaustion of the growth-rate-limiting substrate (g.l.s.) in most culture systems, which led to proposals that the g.l.s. is acting as a repressor of antibiotic production during the early stages of the culture. These proposals have been applied to carbon sources (see Martin \& Demain, 1978) nitrogen sources (e.g. Aharonowitz, 1980) and phosphate sources (Martin, 1976). Although catabolite regulation per se may contribute to the control of specific steps in biosynthetic pathways for secondary metabolites, exhaustion of carbon source will also result in growth rate decay. Our studies using partial cubic spline interpolation for the continuous analysis of growth rate

†Present address: Biotechnology Research, Whitbread Technical Centre, Park Street, Luton LU1 5SR, UK.

¥Present address: Food Science and Technology, University of Reading, Whiteknights, PO Box 226, Reading RG6 2AP, UK.

Abbreviations: CFBC, cyclic fed batch culture; g.l.s., growth-rate-limiting substrate. during transient states suggested that the relationship between growth rate decay and antibiotic production was dependent on whether glucose or nitrate was the g.l.s. (McDermott et al., 1993). This work and subsequent investigations (e.g. Clark et al., 1995; Lynch \& Bushell, 1995) led us to conclude that growth rate decay, rather than relief from substrate-induced repression, is responsible for induction of antibiotic production. Accumulating molecular evidence tends to support this concept (see Chater \& Bibb, 1996).

We have obtained evidence that antibiotic production is associated with a down-regulation of intracellular protein synthesis rate (Wilson \& Bushell, 1995). Induction occurred if a minimum ratio of charged to uncharged tRNA was obtained (when uncharged tRNA accumulated). The ratio was manipulated by changing the g.l.s. or by addition of protein synthesis inhibitors. We speculated that the dependence of production dynamics on the identity of the g.l.s. was a function of affinity between the organism and the substrates studied (Wilson \& Bushell, 1995).

In cyclic fed batch culture (CFBC), which allows growth rate to be manipulated in the absence of substrate exhaustion, we observed a relationship between relative growth rate and erythromycin synthesis rates (Lynch \& Bushell, 1995). Here, we present measurements of protein synthesis rate during an antibiotic-producing CFBC cycle and estimates of values of substrate affinity constants for glucose and nitrate, consistent with our 
hypothesis for physiological control of secondary metabolite production.

\section{METHODS}

Strains and culture media. Saccharopolyspora erythraea NRRL 2338 was used throughout. Antibiotic concentrations were measured routinely by bioassay using a strain of Arthrobacter citreus (GL1) obtained from the Shell Laboratories culture collection, Sittingbourne, Kent, UK. The chemically defined antibiotic production medium for $S$. erythraea contained the following major nutrients $\left(\mathrm{g} \mathrm{l}^{-1}\right.$ in reverse osmosis purified water): glucose $6, \mathrm{NaNO}_{3} 11 \cdot 21$, $\mathrm{KH}_{2} \mathrm{PO}_{4} 3 \cdot 0, \mathrm{~K}_{2} \mathrm{HPO}_{4} 7 \cdot 0$; and the following additional components $\left(\mathrm{g} \mathrm{l}^{-1}\right): \mathrm{MgSO}_{4} .7 \mathrm{H}_{2} \mathrm{O} 0.25, \mathrm{FeSO}_{4} .7 \mathrm{H}_{2} \mathrm{O} 0.025$, $\mathrm{CuCl}_{2} 0.00053, \mathrm{CoCl}_{2} 0.00055, \mathrm{CaCl}_{2} .2 \mathrm{H}_{2} \mathrm{O} 0.0138, \mathrm{ZnCl}_{2}$ $0.0104, \mathrm{MnCl}_{2} 0.0062, \mathrm{Na}_{2} \mathrm{MoO}_{4} 0.0003$. Glucose, nitrate, phosphate and trace solution were autoclaved separately and then mixed. The $\mathrm{pH}$ was adjusted to $7 \cdot 0$ with $5 \mathrm{M} \mathrm{KOH}$ prior to autoclaving.

$S$. erythraea and $A$. citreus were routinely maintained on nutrient agar, and grown in $250 \mathrm{ml}$ baffled Erlenmeyer flasks containing $50 \mathrm{ml}$ medium at $30^{\circ} \mathrm{C}$ on a rotary shaker at 250 r.p.m. A nutrient broth starter medium was inoculated with colonies picked off a plate. After $48 \mathrm{~h}$ agitation, $2 \mathrm{ml}$ was removed and used (S. erythraea) to inoculate preculture flasks containing the defined medium. After $48 \mathrm{~h}$ further incubation, the precultures were used at approximately $5 \%(\mathrm{v} / \mathrm{v})$ as an inoculum for bioreactors.

Bioreactor culture. The Braun Biostat MD bioreactor used had maximum and minimum working volumes of $10.5 \mathrm{l}$ and 4.51 respectively during CFBC (Lynch \& Bushell, 1995). Agitation was provided by disc turbine impellers rotating at 1000 r.p.m. and sterile air was supplied through a sparger. The temperature was controlled at $30^{\circ} \mathrm{C}$. Dissolved oxygen concentration in the bioreactor was monitored with an Ingold polarographic dissolved oxygen electrode and maintained above $80 \%$ of air saturation by varying the airflow rate automatically. The $\mathrm{pH}$ was controlled at $7 \cdot 0$ using automatic additions of $0.1 \mathrm{M} \mathrm{HCl}$ and $0.1 \mathrm{M} \mathrm{NaOH}$. Foaming was eliminated by including $0.01 \%(\mathrm{v} / \mathrm{v})$ Breox FMT30 antifoam (Water Management and Gamlen) in the culture medium.

Medium feeds employed a Braun FE 211 high-precision pistonactuated dosing pump, controlled by microMFCS fermenter control software (Braun) running on a personal computer.

Culture biomass concentration determination. Ten millilitre biomass samples were collected on pre-dried membrane filters (Whatman $0.45 \mu \mathrm{m}$ ). Filtrates were collected and frozen for further assays (erythromycin, vancomycin, glucose, nitrate and phosphate), and the filter was rinsed with distilled water $(3 \times 10 \mathrm{ml})$ prior to drying in a microwave oven (high power, $5 \mathrm{~min}$ ). Dry weights and, hence, biomass concentrations were estimated after cooling and desiccation.

Antibiotic assays. A bioassay employing Artbrobacter citreus was performed using procedures described previously (Huck et al., 1991). Seed cultures and assay plates of $A$. citreus were incubated at $30^{\circ} \mathrm{C}$, and the diameters of the zones of inhibition were recorded after $24 \mathrm{~h}$. Examination of a number of samples using high-performance liquid chromatography (Tsuji \& Goetz, 1978) confirmed that the bioassay corresponded to erythromycin.

Residual glucose, nitrate and phosphate. A glucose-oxidasebased assay kit (Trinder system, Sigma) and a nitrate-
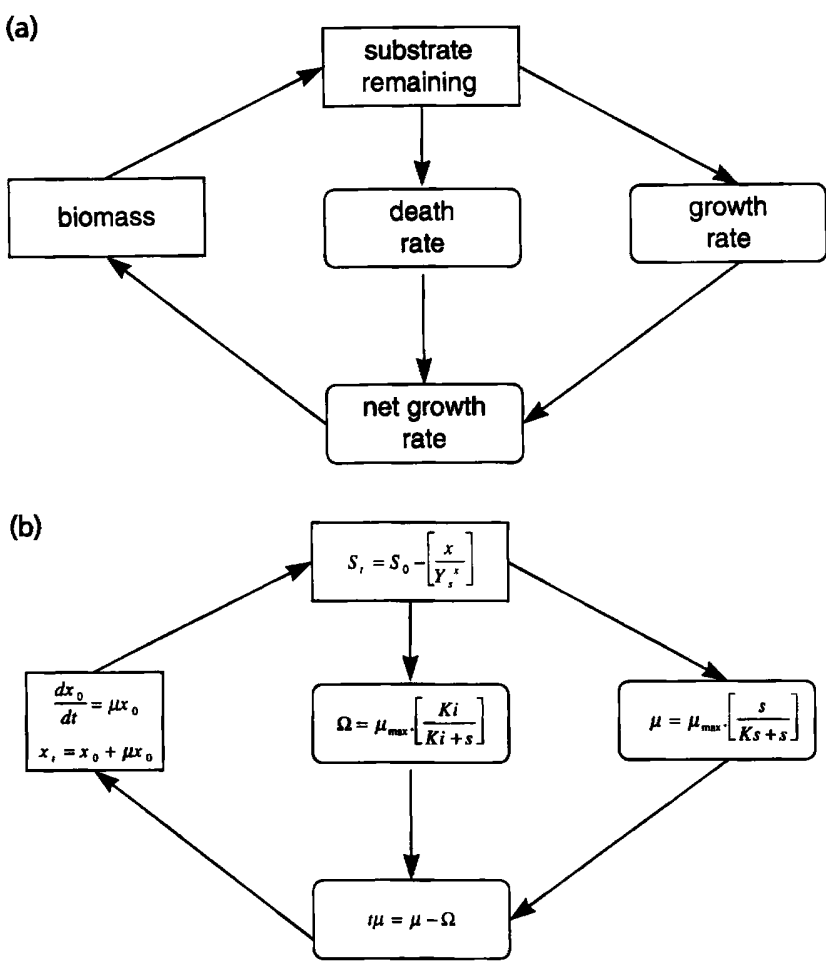

Fig. 1. (a) Components of the model for growth, viability and substrate utilization used to estimate values for $K_{s i}$ (b) equations used to model the components.

reductase-based assay kit (Boehringer Mannheim) were employed. The colorimetric procedure described previously (McDermott et al., 1993) was used for phosphate determination.

Specific biomass and antibiotic production rates. Specific growth rates were calculated using the partial cubic spline interpolation methodology described by us previously (Bushell et al., 1993; McDermott et al., 1993). The idea of using cubic spline interpolation of fermentation data was first proposed by Oner et al. (1986).

Specific rates of protein synthesis. These were estimated by modifying the method of Granozzi et al. (1990) as follows. Radiolabelled leucine solution $\left[25 \mu \mathrm{Ci} \mathrm{ml} l^{-1}\left(925 \mathrm{kBq} \mathrm{ml}^{-1}\right)\right.$; $5 \mathrm{mM}$ ] was added to $15 \mathrm{ml}$ samples of bioreactor culture in $250 \mathrm{ml}$ Erlenmeyer flasks which were shaken at $30^{\circ} \mathrm{C}$. for $25 \mathrm{~min}$. Growth and incorporation were halted by diluting $1 \mathrm{ml}$ samples, taken in duplicate at $5 \mathrm{~min}$ intervals, with $10 \%$ $(\mathrm{w} / \mathrm{v})$ aqueous trichloracetic acid solution $(5 \mathrm{ml})$. After 25 min, samples were filtered (Whatman GF/G sintered glass microfibre filters) and washed with $5 \%(\mathrm{w} / \mathrm{v})$ aqueous trichloracetic acid solution. Radioactivity was estimated in $4 \mathrm{ml}$ scintillation fluid (Optiphase Safe, LKN).

Determination of substrate affinities. Samples from bioreactor cultures were taken at $2 \mathrm{~h}$ intervals and biomass and g.l.s. concentrations determined. Estimates of affinity constant values $\left(K_{\mathrm{s}}\right)$ for glucose and nitrate were obtained using the S B Modelmaker (S B Technology) package. A model was constructed (Fig. 1) assuming that biomass accretion kinetics could be described in terms of the Monod growth rate function $(\mu)$ and a death rate function $(\Omega)$ of Bree et al. (1988). The package estimated the most probable values of maximum 
specific growth rate $\left(\mu_{\max }\right)$ and $K_{\mathrm{s}}$ required to fit the experimental data for biomass and glucose concentrations to the model equations.

Reproducibility and replication of experiments. All experimental data were obtained from single cultures. Experiments were carried out in triplicate to ensure that the trends and relationships observed in the culture parameters measured were reproducible. Individual assays were replicated fourfold except where stated. Experiments were rejected where a chisquared test indicated significant differences between replicates. Where error bars are not shown, they were too small to be visible on the figures presented.

\section{RESULTS AND DISCUSSION}

\section{Batch culture production dynamics}

Erythromycin production was observed under carbonand nitrogen-limited conditions (Fig. $2 a, c$ ). The frequency of sampling in this study was low, due to the requirements for large samples for the protein synthesis assay. Although this decreases the confidence that can be placed on the interpolated curves, growth and antibiotic production kinetics were consistent with previous findings, which included studies where very frequent sampling was possible (McDermott et al., 1993; Clark et al., 1995; Wilson \& Bushell, 1995). Analysis using partial cubic spline interpolation indicated that the specific antibiotic production rate peaked after the specific growth rate in carbon limited culture (Fig. 2b). This is the classic growth-dissociated production pattern associated with secondary metabolism. The peaks in growth and production rates coincided in the nitrogenlimited medium, exhibiting an apparent growthassociated production pattern in this culture (Fig. 2 b). These patterns are consistent with our previous findings (McDermott et al., 1993; Clark et al., 1995; Wilson \& Bushell, 1995; Lynch \& Bushell, 1995).

\section{Correlation between protein synthesis and antibiotic production rates in batch culture}

The intracellular protein synthesis rate decreased after having reached a maximum at approximately $37 \mathrm{~h}$ in carbon-limited culture (Fig. 2b). As in our previous study (Wilson \& Bushell, 1995), the increase in the antibiotic production rate coincided with the period when the protein synthesis rate decreased significantly (to approximately half its peak value) and the specific growth rate was declining (Fig. 2b). Kinetics in nitrogenlimited batch culture also supported our previous findings. The protein synthesis rate was half that observed in carbon-limited culture and the increase in
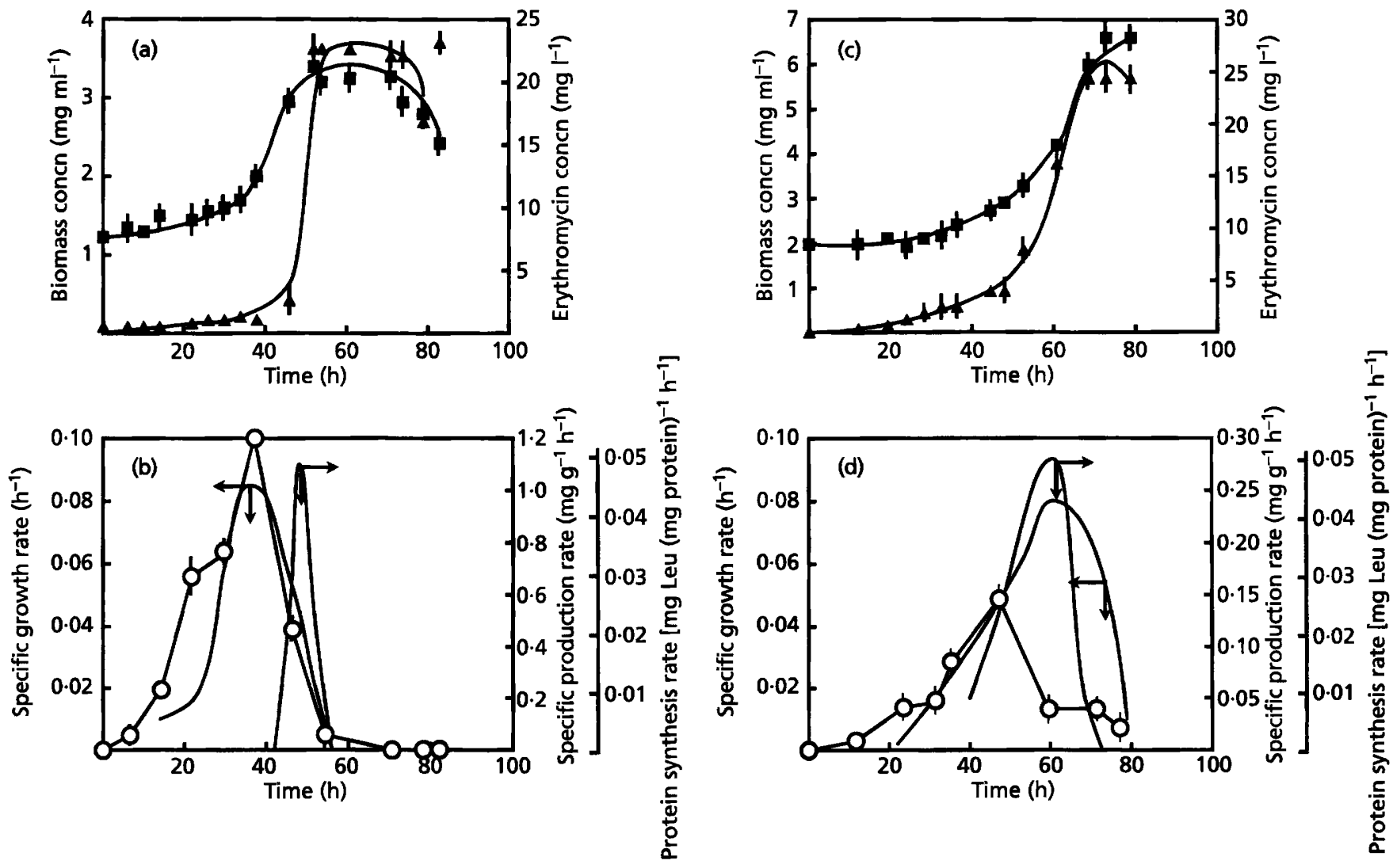

Fig. 2. Results from glucose-limited $(a, b)$ and nitrate-limited (c, d) cultures in bioreactors. (a, c) $\square$, Biomass concentration; $A$, antibiotic concentration; (b, d) continuous line, left axis referenced, interpolated specific growth rate; continuous line, right axis referenced, interpolated specific antibiotic production rate; $O$, protein synthesis rate. 


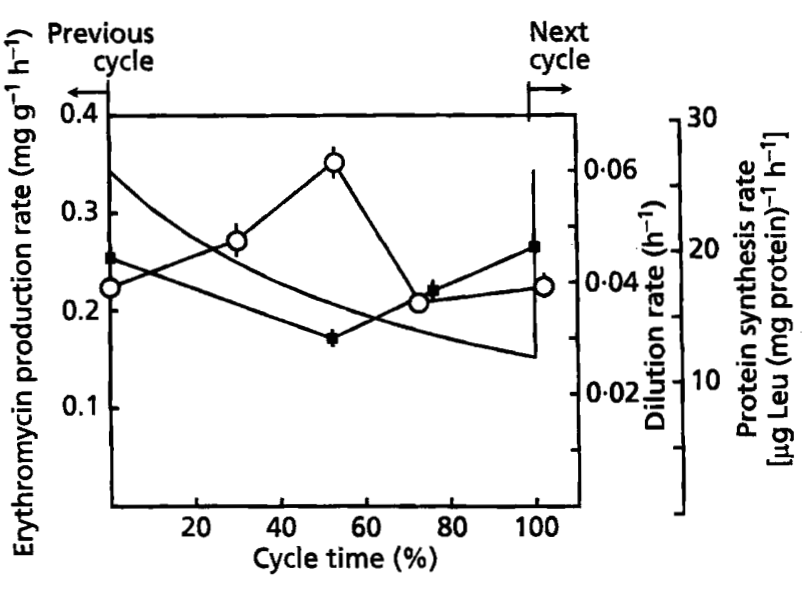

Fig. 3. Dilution rate (curve), protein synthesis rate $(O)$ and erythromycin production rate $(\square)$ in cyclic fed batch culture.

antibiotic production rate paralleled the increase in growth rate (Fig. $2 \mathrm{~d}$ ).

\section{Correlation between protein synthesis and antibiotic production rates in CFBC}

The antibiotic yields obtained from this wild-type strain in simple batch culture with synthetic media are considerably lower than those obtained in commercial production processes, where sophisticated feeding regimes are applied to mutant organisms growing in complex media. We developed CFBC as a means of increasing antibiotic production by imposing a constant down-regulation of growth rate on the culture (Lynch \& Bushell, 1995) when we reported phases of decreased and increased antibiotic production rate during different phases of the CFBC cycle. During the experiment reported in the present paper, we also measured the intracellular protein synthesis rate. The protein synthesis rate increased at the start of each cycle, then decreased, before increasing again (Fig. 3). As in batch culture, the antibiotic synthesis rate had an inverse relationship with protein synthesis rate. It increased during the phase of decreasing protein synthesis rate and declined only when the protein synthesis rate increased.

We have suggested that a critical decrease in protein synthesis rate acts as a trigger for secondary metabolite production (Wilson \& Bushell, 1995). In all cultures where antibiotic was detected during that study, onset of antibiotic production coincided with minimal protein synthesis rate. Further investigation in $S$. erytbraea and Streptomyces hygroscopicus cultures indicated that this corresponded to the minimum ratio of charged to uncharged tRNA, i.e. when uncharged tRNA accumulated (Wilson \& Bushell, 1995). Results obtained in the present paper indicate that the benefits in antibiotic yield provided by the CFBC technique derive from its ability to induce a down-shift in protein synthesis rate (Fig. 3), presumably leading to the requisite accumulation of uncharged $t R N A$.
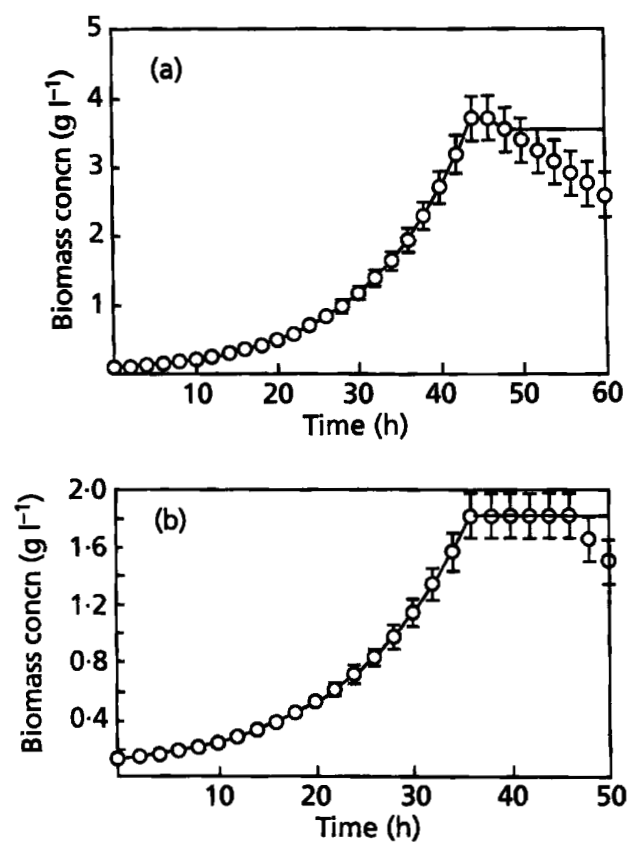

Fig. 4. Experimental data $(O)$ and data predicted by the model (lines) for (a) glucose-limited and (b) nitrate-limited batch culture data.

\section{Relevance of production dynamics to an ecological role for secondary metabolites}

Secondary metabolites may function in the environment as inhibitors of competitor organisms or as signalling compounds, such as mating factors (Roth et al., 1986). In either case, a means of sensing the presence of other micro-organisms is needed if the compounds are not to be produced constitutively (and, therefore, wastefully). The arrival of a new microbial cell into a micro-habitat already occupied by a potential secondary metabolite producer would result in a transient decrease in nutrient availability if both were capable of utilizing the same nutrients to the same extent. This would result in a decrease in growth rate, thus providing a trigger for secondary metabolite production. Such a sensing mechanism, based entirely on nutrient uptake rate, would ensure secondary metabolite production for inhibition of competitors or signalling to potential mating strains, only when a significant change in the assimilation rate of the growth-limiting nutrient occurs. In the competition scenario, this would be the only circumstance when antibiotic production would be needed.

\section{Effect of substrate affinity on production dynamics}

During a batch liquid culture, in which the organism has a high affinity for the g.l.s., the growth rate remains close to its maximum value until the concentration of the substrate falls to a value commensurate with the substrate affinity $\left(K_{\mathrm{s}}\right)$ value. When this point is reached, the growth rate falls very rapidly. This rapid decrease coincides with the onset of antibiotic production during 


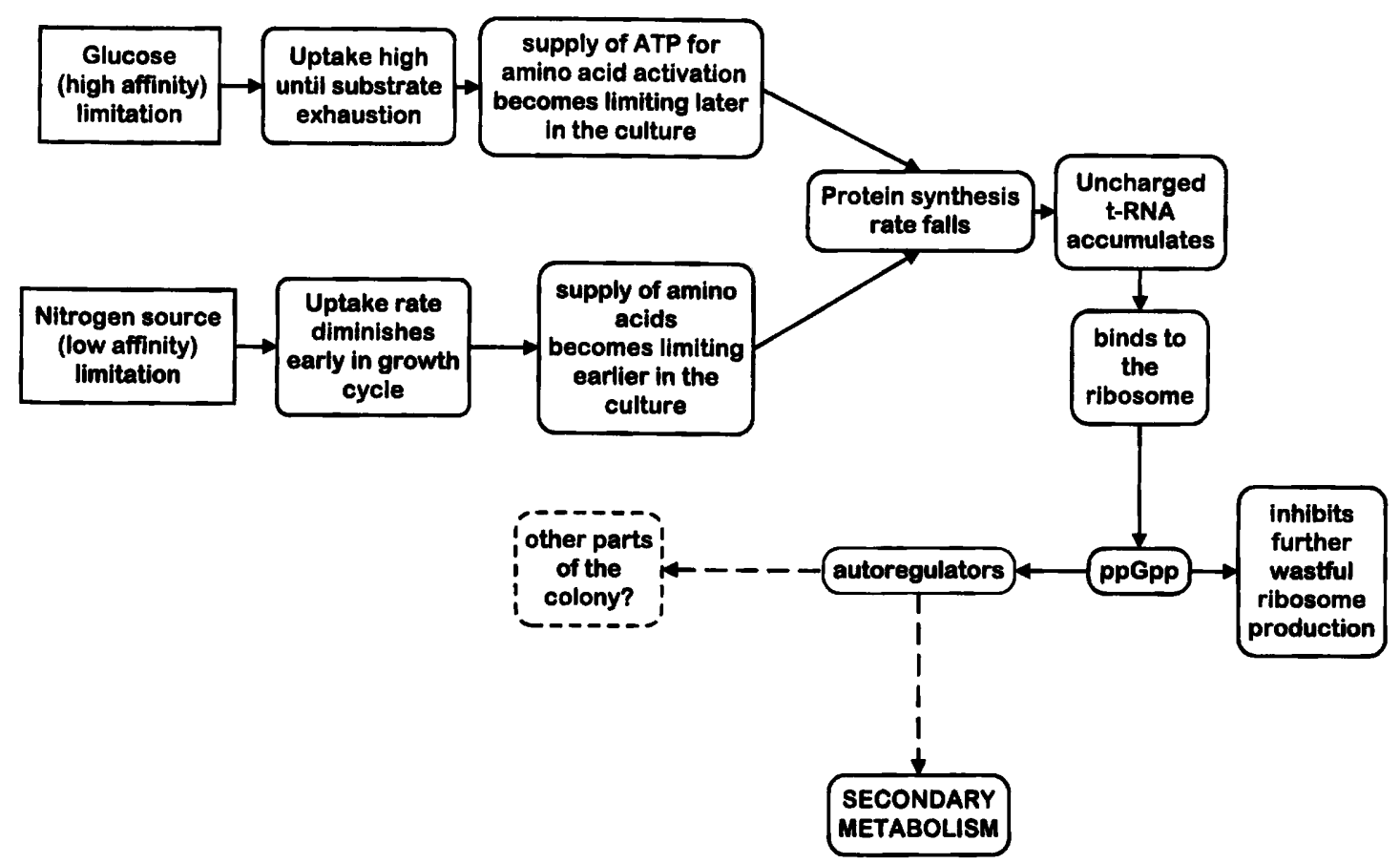

Fig. 5. Physiological model based on the literature and results discussed in this paper.

late exponential phase (i.e. 'secondary' metabolism) in carbon-limited culture. In nitrogen-limited culture the organism has a low affinity for the g.l.s. Nitrogen exhaustion, therefore, occurs gradually rather than as a transient event. Protein synthesis rate and tRNA charging are sensitive to the magnitude and rate of change of extracellular nutrient availability (Koch, 1980) and are, therefore, reasonable candidates for key components of the cascade leading to antibiotic production.

We estimated values for substrate affinity constant values for glucose and sodium nitrate, using the nonlinear modelling technique approach described (Methods, 'Determination of substrate affinities'), in batch culture. The growth curves could be modelled accurately until late stationary phase (Fig. 4). Our estimates indicated that the affinity for nitrate $\left(K_{\mathrm{s}}=\right.$ $7.8 \mathrm{mM}, \pm 4 \times 10^{-2}$ ) was, indeed, lower than that for glucose $\left(\bar{K}_{\mathrm{s}}=7.7 \times 10^{-3} \mathrm{mM}, \pm 4 \times 10^{-5}\right)$, providing support for the physiological control model shown in Fig. 5.

\section{Elements of the model for physiological control of secondary metabolism}

An essential role for butyrolactones such as A factor in the cascade has also been proposed (Beppu, 1992). This requires investigation to determine whether they are an essential part of the sequence of compounds required to initiate secondary metabolism or simply act as diffusible signal factors (Fig. 5), whose function is to initiate antibiotic production in parts of the colony, remote from that in which the growth rate down-regulation event has taken place.
Measurements of the substrate affinities, presented in this paper, support the concept of two alternative regulatory cascades between g.l.s. (glucose and nitrogen source) limitation and protein synthesis rate, proposed in Fig. 5. This provides a hypothesis that can account for the growth-associated and growth-dissociated production kinetics observed under, respectively, glucose and nitrate limitation. The pattern of protein synthesis rate observed during a CFBC cycle, also reported here, provides evidence that down-regulation in protein synthesis rate results in antibiotic production, a concept first developed in a previous study (Wilson \& Bushell, 1995) during which it was established that this downregulation only effects initiation of secondary metabolism if it results in a significant increase in uncharged tRNA. This observation supports the assertion of Ochi (1988) and Bibb (1996) that ppGpp probably plays a crucial role in initiation of antibiotic synthesis, since accumulation of uncharged tRNA initiates production of ppGpp (leading to the stringent response; Lamond \& Travers, 1985). However, other groups (Takano et al., 1992; Bascaran et al., 1991) claim antibiotic production in the absence of elevated ppGpp levels, so there is clearly a requirement for more work in this area.

The physiological control model, presented here is compatible with the molecular control model proposed recently by Bibb (1996).

\section{REFERENCES}

Aharonowitz, Y. (1980). Nitrogen metabolite regulation of antibiotic biosynthesis. Annu Rev Microbiol 34, 209-233. 
Bascaran, V., Sanchez, L., Hardisson, C. \& Brana, A. F. (1991). Stringent response and initiation of secondary metabolism in Streptomyces clavuligerus. J Gen Microbiol 137, 1625-1634.

Beppu, T. (1992). Secondary metabolites as chemical signals for cellular differentiation. Gene 115, 159-165.

Bibb, M. J. (1996). The regulation of antibiotic production in Streptomyces coelicolor A3(2). Microbiology 142, 1335-1344.

Bree, M. A., Dhurjati, P., Georghegan, R. F. \& Robnett, B. (1988). Kinetic modelling of hybridoma cell growth and immunoglobulin production in a large-scale suspension culture. Biotechnol Bioeng 32, 1067-1072.

Bushell, M. E., Bell, S. L., Scott, M. F., Snell, K., Speir, R. E., Wardell, J. N. \& Sanders, P. G. (1993). A three-phase pattern in growth, monoclonal antibody production and metabolite exchange in a hybridoma bioreactor culture. Biotechnol Bioeng 42, 133-139.

Chater, K. F. \& Bibb, M. J. (1996). Regulation of bacterial antibiotic production. In Products of Secondary Metabolism (Biotechnology, vol. 6). Edited by $H$. Kleinkauf \& $H$. von Dohren. Weinheim: VCH (in press).

Clark, G. J., Langley, D. \& Bushell, M. E. (1995). Oxygen limitation can induce microbial secondary metabolite formation: investigations with miniature electrodes in shaker and bioreactor culture. Microbiology 141, 663-669.

Demain, A. L., Aharonowitz, Y. \& Martin, J.-F. (1983). Metabolic control of secondary biosynthetic pathways. In Biochemistry and Genetic Regulation of Commercially Important Antibiotics, pp. 49-72. Edited by L. C. Vining. Reading, MA: Addison-Wesley.

Granozzi, C., Billetta, R., Passantino, R., Sallazo, M. \& Puglia, A. M. (1990). A breakdown in macromolecular synthesis preceding differentiation in Streptomyces coelicolor (A3)2. J Antibiot 29, 713-716.

Huck, T. A., Porter, N. \& Bushell, M. E. (1991). Positive selection of antibiotic-producing soil isolates. J Gen Microbiol, 137, 2321-2329.

Koch, A. L. (1980). The inefficiency of ribosomes functioning in Escherichia coli growing at moderate rates. J Gen Microbiol 116, 165-171.

Lamond, A. I. \& Travers, A. A. (1985). Stringent control of bacterial transcription. Cell. 41, 313-334.
Lynch, H. C. \& Bushell, M. E. (1995). The physiology of erythromycin biosynthesis in cyclic fed batch culture. Microbiology 141, 3105-3111.

McDermott, J. F., Lethbridge, G. \& Bushell, M. E. (1993). Estimation of the kinetic constants and elucidation of trends in growth and erythromycin production in batch and continuous cultures of Saccharopolyspora erythraea using curve fitting techniques. Enzyme Microb Technol 15, 657-663.

Martin, J. F. (1976). Phosphate regulation of gene expression in candidicin biosynthesis. In Microbiology-1976, pp. 548-552. Edited by D. Schlessinger. Washington, DC: American Society for Microbiology.

Martin, J. F. \& Demain, A. L. (1978). Control of antibiotic biosynthesis. Microbiol Rev 44, 230-251.

Ochi, K. (1988). Nucleotide pools and stringent response in regulation of Streptomyces differentiation. In Proceedings of the $7 \mathrm{th}$ International Symposium on the Biology of Actinomycetes, pp. 330-337. Edited by Y. Okami, T. Beppu \& H. Ogawara. Tokyo: Japan Scientific Societies Press.

Oner, M. D. Erickson, L. E. \& Young S. S. (1986). Utilisation of spline functions for smoothing fermentation data and for estimation of specific rates Biotechnol Bioeng, 28, 902-918.

Roth, J., Leroith, D., Collier, E. S., Watkinson, A. \& Lesniak, M. A. (1986). The evolutionary origins of intercellular communication and the Maginot Lines of the mind. Ann NY Acad Sci, 463,1-11.

Takano, E., Gramajo, H. C., Stauch, E., Andres, N., White, J. \& Bibb, M. J. (1992). The stringent response in Streptomyces coelicolor A3(2). Mol Microbiol 6, 2797-2804.

Tsuji, K. \& Goetz, J. F. (1978). High performance liquid chromatographic determination of erythromycin. J Chromatogr 147, 359-367.

Wilson, G. C. \& Bushell, M. E. (1995). The induction of antibiotic synthesis in Saccharopolyspora erythraea and Streptomyces hygroscopicus by growth rate decrease is accompanied by a down-regulation of protein synthesis rate. FEMS Microbiol Lett $129,89-96$.

Received 12 June 1996; revised 12 September 1996; accepted 23 September 1996. 